\title{
Guided Acoustic Waves for Liquid Property Measurement
}

\author{
Rautenberg, Jens; Bause, Fabian; Henning, Bernd \\ University of Paderborn, Department of Electrical Engineering and Information Technology \\ Measurement Engineering Group, Warburger Straße 100, 33098 Paderborn, Germany \\ E-Mail: Rautenberg@emt.uni-paderborn.de
}

This contribution will give a short survey on acoustic waveguides that are used for the measurement of liquid properties. As guided acoustic waves range from the $\mathrm{mm}$-scale to the $\mathrm{km}$-scale, they are used in lots of scientific disciplines like seismology, geophysics, non-destructive testing, biological and chemical analysis or process measurement engineering. One phenomenological way of classifying these waveguides will be developed, always considering the special characteristics like in- and out-of-plane displacements of the waveguide's boundary as well as single mode, multimode or bulk mode operation. Later on, this article will focus on the modelling, simulation and measurement of multimode wave propagation in acoustic waveguides.

\section{Rheologic and acoustic properties of a liquid}

The acoustic coupling to a liquid as well as the wave propagation within a medium highly depend on physical and rheological properties of the sensor system. Focusing on the bulk properties of a medium, the one-dimensional rheologic equation of state is a good starting point to gain an insight into the denoted interrelationship. If the amplitudes are small and the liquid is a Newtonian fluid, the constitutive equations for stress $\sigma$ and strain $\varepsilon$ are

$$
\left.\begin{array}{ll}
\sigma=K \cdot \varepsilon & \text { (Elasticity, Hook's law) } \\
\sigma=\eta_{\mathrm{s}} \cdot \dot{\varepsilon} & \text { (Viscoelasticity, Newton's law) }
\end{array}\right\} \Rightarrow \sigma=K \cdot \varepsilon+\eta_{\mathrm{s}} \cdot \dot{\varepsilon}
$$

with the bulk modulus of compressibility $K$ and the shear viscosity $\eta_{\mathrm{s}}$ of the liquid. Therewith, the onedimensional equation of motion for particle velocity $\dot{\varepsilon}$ in the time- and frequency-domain is [1]:

$$
\left(K+j \frac{4}{3} \omega \eta_{\mathrm{S}}\right) \frac{\partial^{2} \dot{\varepsilon}(z)}{\partial z^{2}}+\rho_{0} \omega^{2} \dot{\varepsilon}(z)=0 \Rightarrow \underline{K} \frac{\partial^{2} \dot{\varepsilon}(z)}{\partial z^{2}}+\rho_{0} \omega^{2} \dot{\varepsilon}(z)=0
$$

Therein $\underline{K}(\omega)=K(\omega)+j 4 / 3 \cdot \eta_{\mathrm{S}}(\omega)$ is the complex valued compressive modulus. Its real part $K^{\mathrm{R}}(\omega)$ corresponds to the storage modulus, whereas the imaginary part $K^{1}(\omega)=4 / 3 \cdot \eta_{\mathrm{S}}(\omega)$ represents the viscous losses in the material. The assumption of causality (i.e. no condensation response without a causal pressure stimulus) leads to the acoustic Kramers-Kronig-Relations [2]:

$$
K^{\mathrm{R}}(\omega)=\frac{2}{\pi} \mathrm{P} \int_{0}^{\infty} K^{\mathrm{I}}\left(\omega^{\prime}\right) \cdot \frac{\omega^{\prime}}{\omega^{2}-\omega^{\prime 2}} d \omega^{\prime} \quad K^{\mathrm{I}}(\omega)=-\frac{2 \omega}{\pi} \mathrm{P} \int_{0}^{\infty} K^{\mathrm{R}}\left(\omega^{\prime}\right) \cdot \frac{1}{\omega^{2}-\omega^{\prime 2}} d \omega^{\prime}
$$

Due to the singularity at $\omega^{\prime}=\omega$, the integral has to be solved as a principal value, denoted with $\mathrm{P}$. The real and imaginary parts of the frequency dependant bulk modulus interrelate to each other and each material law has to fulfill these equations. This plays a decicive role for the acoustic wave propagation in an absorptive medium. With the complex valued wavenumber $\xi=\omega c^{-1}(\omega)+j \alpha(\omega)$ and also a complex sound velocity $\underline{c}(\omega)=\sqrt{\underline{K}(\omega) / \rho_{0}}$ it is possible to expand equation set (3) into a lokal form around a centre frequency $\omega_{0}$. At this frequency the speed of sound is $c_{0}$ and the density $\rho_{0}$ of the liquid is assumed to be constant:

$$
\alpha(\omega)=\frac{1}{c(\omega)} \cdot \frac{\omega \cdot \mathrm{K}_{\mathrm{V}}(\omega)}{\sqrt{4-\mathrm{K}_{\mathrm{V}}(\omega)^{2}}} \quad c(\omega) \approx c_{0}+\frac{2}{\pi} c_{0}^{2} \cdot \int_{\omega_{0}}^{\omega} \frac{\alpha\left(\omega^{\prime}\right)}{\omega^{\prime 2}} d \omega^{\prime}
$$

In this form $\mathrm{K}_{\mathrm{V}}(\omega)=4 \eta_{\mathrm{S}}(\omega) / 3 K(\omega)$ is the ratio of imaginary and real part of the complex bulk modulus. Therewith, it is obvious that there is a causal relation between the characteristics of sound propagation and physical or rheological material behaviour as it is expressed in $\mathrm{K}_{\mathrm{v}}(\omega)$. So, if both spectra (or parts of it) are known, it is possible to match the frequency dependant material properties to equation (4a) and therwith to find the variable of interest just from acoustic transmission measurements through the analyte.

Apart from these bulk characteristics, the liquid also affects the wavepropagation in a solid by the solidliquid interface, because it changes the boundary conditions. So let's consider a plane wave in a solid half 
space adjoining the predescribed liquid. Depending on the particle movement at the solid side of the boundary, different properties of the liquid affect the amplitudes of the reflected wave. First, considering the out-of-plane components of the incident wave, the main property is the real part of the liquids specific acoustic impedance $\mathfrak{R}\{\underline{Z}(\omega)\}=\mathfrak{R}\left\{\rho_{0} \cdot \underline{c}(\omega)\right\} \approx \rho_{0} \cdot c(\omega)$. This simplification is valid because there are no considerable volume viscous losses in the liquid, and the out-of-plane components only excite longitudinal waves. The more the impedance matches the longitudinal wave impedance $Z_{\mathrm{L}}=\rho_{\mathrm{S}} \cdot c_{\mathrm{L}}$ of the solid, the better the out-of-plane components leak into the liquid. The amplitude transmission factor $T^{\mathrm{R}}$ is:

$$
T^{\mathrm{R}}=\frac{2 \rho_{0} c(\omega)}{\rho_{0} c(\omega)+\rho_{\mathrm{s}} c_{\mathrm{L}}}
$$

Due to the fact that the liquid density $\rho_{0}$ is involved this is known as mass effect. Secondly, considering the in-plane components of the incident wave, these components only loose energy to the liquid if there is a significant imaginary part $\mathfrak{I}\{\underline{Z}(\omega)\} \approx\left(2 \eta_{\mathrm{s}}\right) /\left(3 \rho_{0} c(\omega)\right)$ of the specific impedance. This simplification is valid as the in-plane components only exite an inhomogeneous wave in the liquid. With the transversal specific impedance $Z_{\mathrm{T}}=\rho_{\mathrm{s}} \cdot c_{\mathrm{T}}$ of the solid, the transmission factor $T^{\mathrm{I}}$ is

$$
T^{\mathrm{I}}=\frac{4 \eta_{\mathrm{s}}}{2 \eta_{\mathrm{s}}+3 \rho_{0} c(\omega) \rho_{\mathrm{s}} c_{\mathrm{T}}}
$$

This effect is dominated by the viscosity of the liquid. Therewith it is possible to decompose massproportional and viscosity-proportional damping of a wave by a proper selection of the used wavetypes. In both cases it is essential to know both, the initial amplitudes and the sound velocity at the operation frequency.

\section{Classification of waveguide sensors}

As guided acoustic waves range from the $\mathrm{mm}$-scale to the $\mathrm{km}$-scale, it is no surprise that they are used in lots of different scientific disciplines like seismology, geophysics, non-destructive testing, biological and chemical analysis or process measurement engineering (Fig. 1). But the basic equations that are used for modelling wave propagation are always the same. Additionally, the mechanisms of interaction between liquid and acoustic wave can all be matched to one of the effects that are described in Eq. $3-6$.

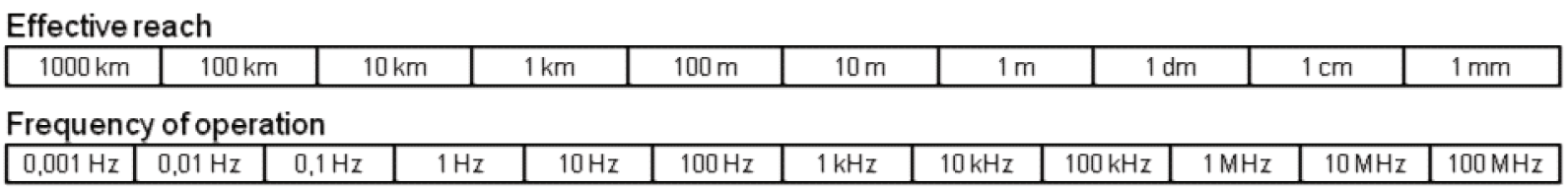
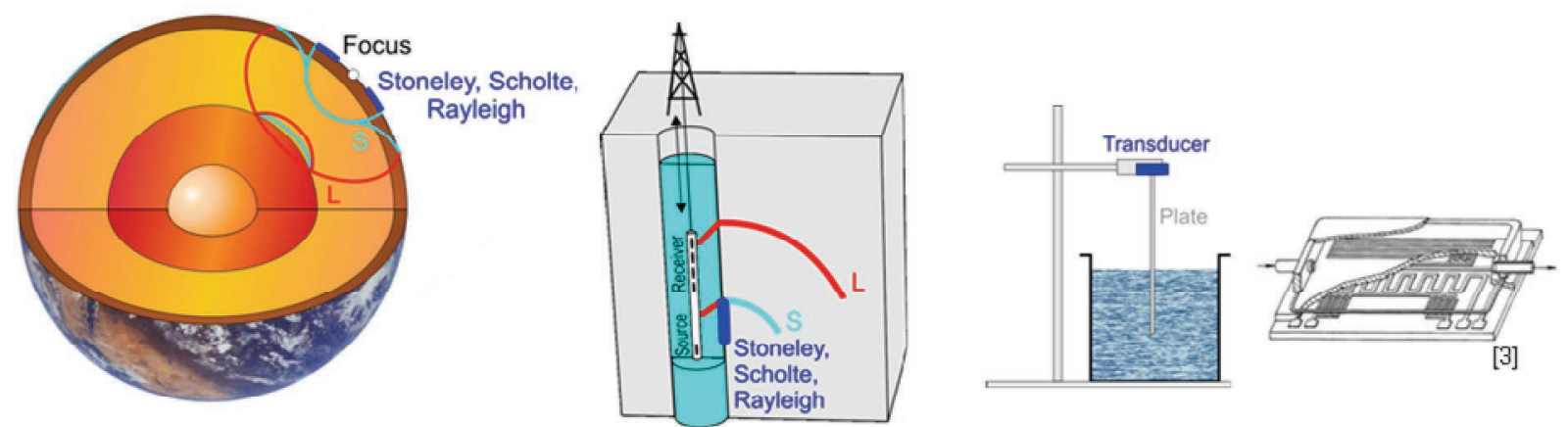

Figure 1: Guided waves in different scales: There are lots of applications, differing by the frequency of operation and therewith by reach of the waves and size.

Apart from the scale, the two described mechanisms of influencing sound propagation lead to a phenomenological way of classifying acoustic waveguides.

\section{Solid/liquid interface sensors:}

a. In-plane displacements: With Love- or Scholte-waves it is possible to sense mainly viscosity and there are a lot of realizations in different fields of applications at each scale [3-8].

b. Out-of-plane displacements: Guided waves with pure out-of-plane displacements are nonexistent. Therefore most of acoustic impedance measurement systems are not based on waveguides [9].

c. Mixed displacements: There are lots of waves like the leaky Rayleigh- or leaky Lamb-waves, whose particle movements at the interface are both in- and out-of-plane. Therewith both 
properties, density and viscosity influence the amplitudes of the guided waves. This is why applications are seldom, one example is given in [10].

\section{Pure liquid waveguides:}

a. Single mode operation: Most of ultrasonic flowmeters are designed in such a way that higher modes don't interfere time-of-flight measurements. The additional measurement of sound velocity allows simple classification of liquids and even concentration measurements.

b. Multimode operation: When the diameter of the waveguide increases the number of propagating modes increases, too. Modelling and sound velocity measurement get more complicated. but there are some approaches to sense both, modespecific times-of-flight and amplitudes [11, 12].

c. Bulk wave operation: Here, the waveguide diameter is large in comparison to the wavelength in the liquid and a wave package travels zig-zag through the waveguide. Therewith very precise sound velocity measurements are possible [13].

\section{Modelling and simulation}

Most of the interface sensing waveguide systems are driven in such a way that only one specific mode is excited. This can be done by adopting the geometry of the waveguide to the frequency of operation and geometry of the transducer or vice versa. A basis for a proper sensor design as well as simulation of wave propagation are the dispersion relations (Fig. 2) that can be calculated by Waveguide FEM or the Global Matrix Method for instance [14-16]. Therin it is also possible to consider the modified boundary conditions at the outer wavegguide surface due to the adjoining liquid medium. The dispersion relations are a survey of solutions of the frequency equation. In addition, each solution corresponds to one specific shape of strains or displacements in the cross-section of the waveguide. Furthermore, the velocityfrequency plots show a geometry dependent dispersion. If the material dispersion (Eq. 4) should be evaluated for liquid characterization it is essential to take also the waveguide dispersion into account.
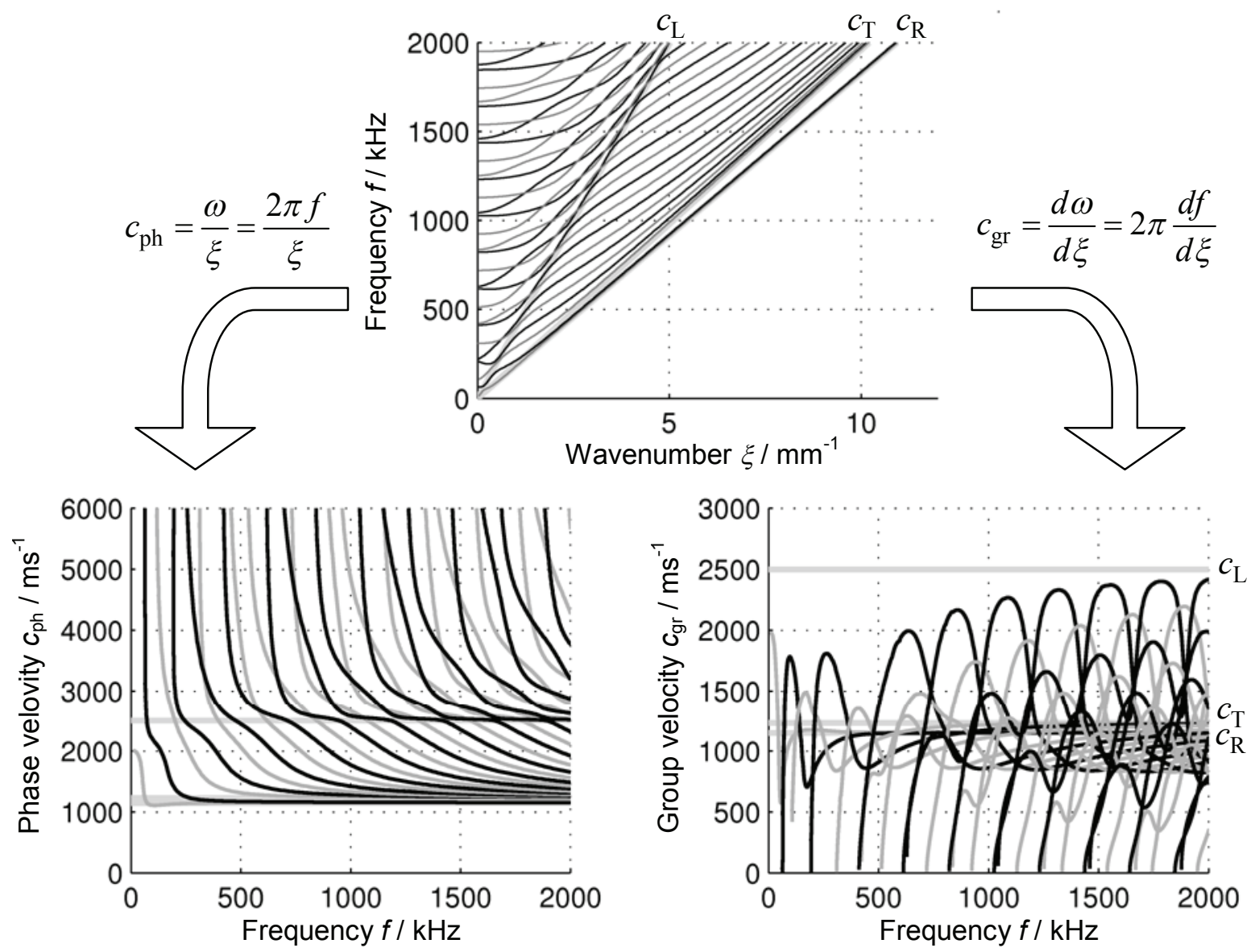

Figure 2: Different types of graphic representation for the dispersion relations for a hollow cylinder (Black: Symmetric modes w.r.t. the neutral axis; Gray: Antisymmetric modes) 
In most of the singlemode operating systems only small wavenumbers are considered (lower left part of frequency-wavenumber plot in Fig.2), whereas in multimode operation some tens of modes are part of wave propagation. Taking also the shape of the modespecific strains into account it is possible to expand any acoustic excitation into its modal components and to propagate them through the waveguide to any other location, even if the propagation media are attenuative. The modal expansion is similar to a Fourier expansion, but the sinusoidal basis function set is replaced by the modal shape functions, which are orthogonal but not necessarily complete. This is why a least squares approach is a good choice for amplitude determination. Figure 3 demonstrates this kind of modal expansion for a hollow cylinder under the assumption of pure normal stress at the beginning of the waveguide.
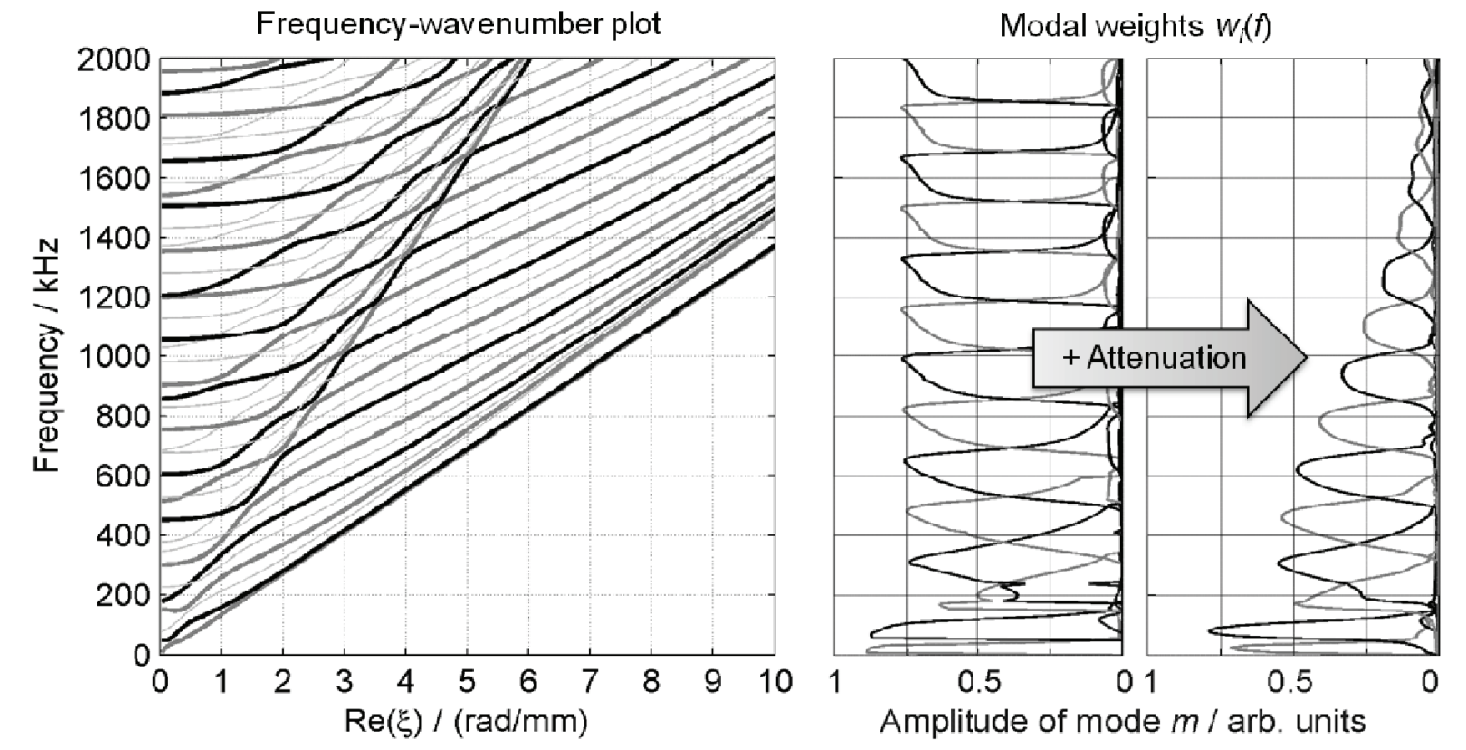

Figure 3: Dispersion relations and modal weights for an axial symmetric waveguide (light gray: Antisymmetric modes; solid lines: Symmetric modes)

It is obvious that only up to approximately three modes contribute to the sound propagation at each frequency. These main components all belong to symmetric modes, caused by the predefined excitation. At this step the frequency dependent absorption as well as material specific and geometric dispersion can be considered, resulting in an acoustic transfer function $G(\mathrm{j} \omega)$. Therewith it is possible to simulate the transmission of an acoustic excitation to any other place in the waveguide. The procedure of recombination for the hollow cylinder, whose expansion is shown in Fig. 3, is demonstrated in Fig. 4. Analytical simulation and measurement are in good agreement.

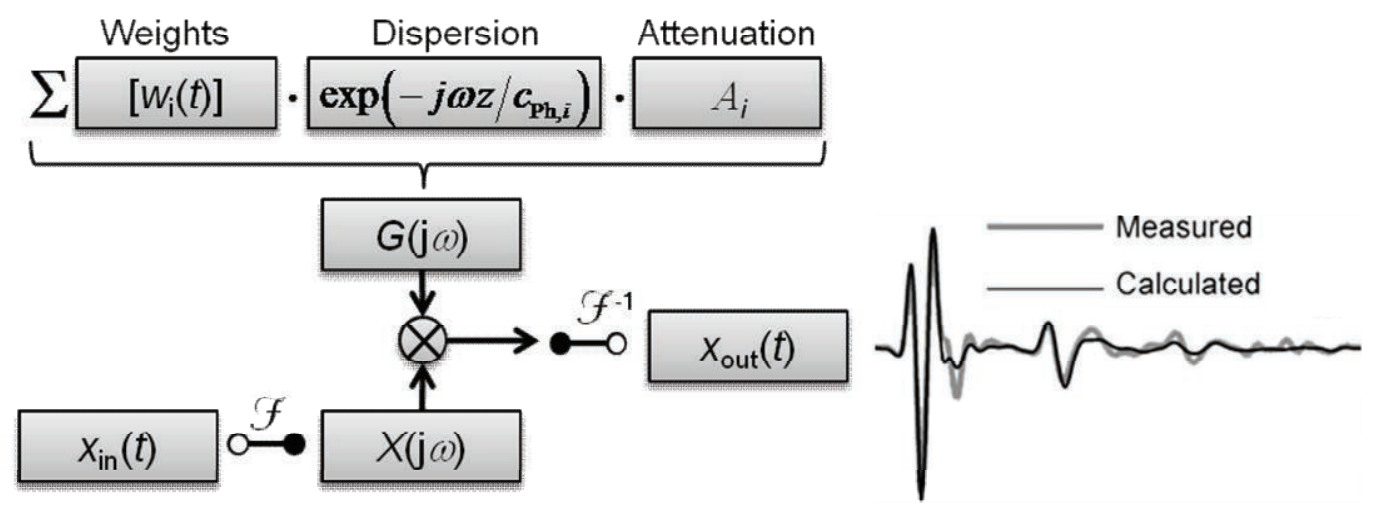

Figure 4: Simulation scheme and one simulation result in comparison to measurement

\section{Measurement of dispersion relations}

Finally the measurement of waveguide properties shall be discussed. There are different ways to measure the dispersion relations and, as so often, it is a trade-off between information content of the measured variables and the technical demands or costs. The most significant method is to measure the 
out-of-plane displacements as a function of time and space at the bounds at the end of the waveguide. A two-dimensional Fast Fourier Transform (2D-FFT) is a good approximation for the frequencywavenumber plot [17].
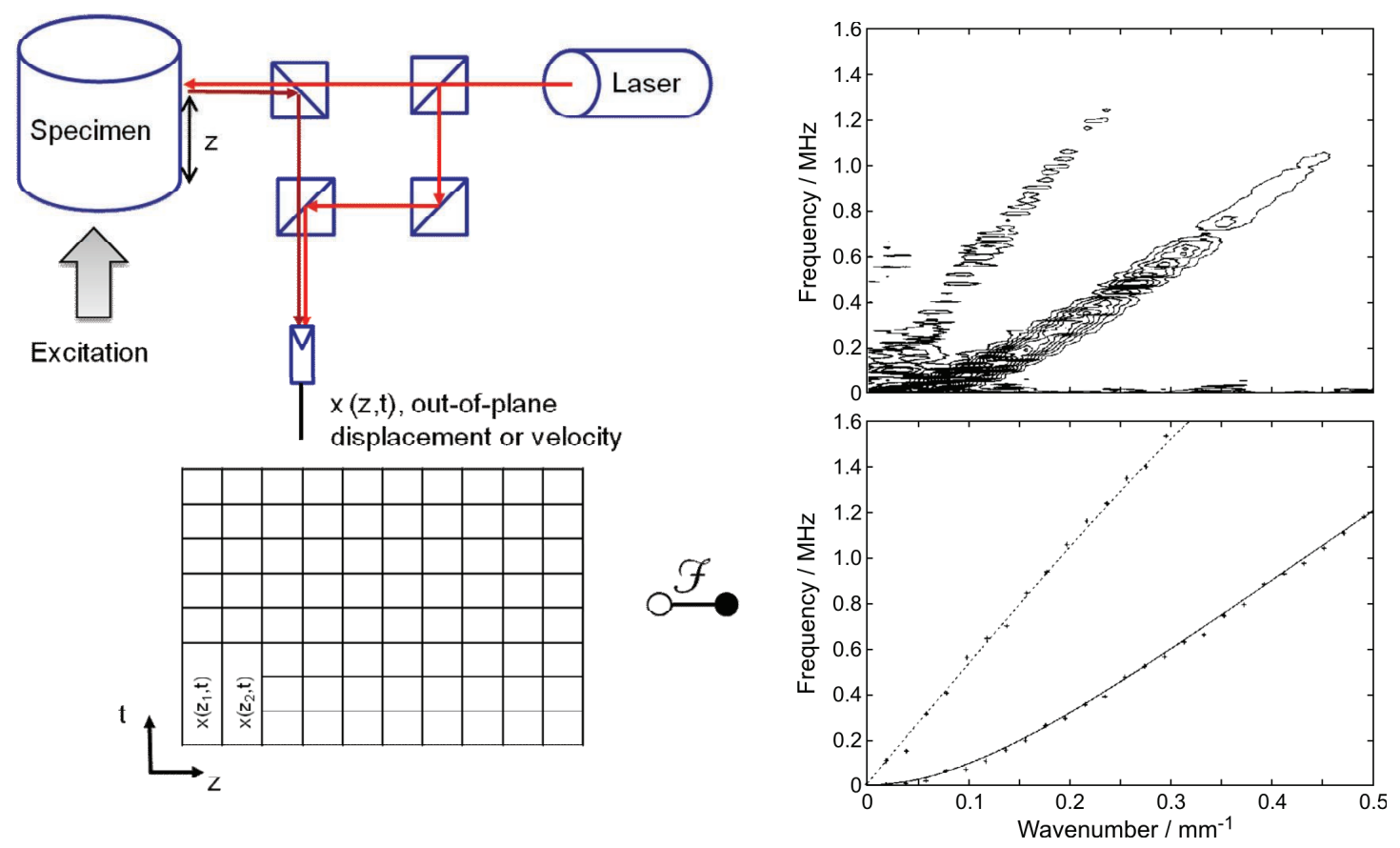

Figure 5: Measurement setup and one result from D. Costley e.a. in [17, Fig. 3] for a $1.016 \mathrm{~mm}$ thick aluminium plate; both Lamb waves are identified by marking the local extrema of the 2D-FFT result

Less expensive but also less meaningful is the sonagram analysis of a single detected signal at the end of the waveguide. The Fourier transforms of the windowed signal are plotted as a function of time while the window is also time-shifted (Fig. 6). The general problem is, that the size of the window determines the uncertainty in the time- and frequency-domain respectively. But if additional information of the window as well as the delay between two consecutive echoes is taken into account it is possible to sharpen the sonagram by an iterative deconvolution algorithm for instance [11]. The first lokal maxima are then a good guess for the modespecific group delays whereas the following maxima indicate bandgaps.

A very low cost solution is the model based Principal Component Analysis (PCA) [12]. In this approach it is assumed that there are analytic eigen-signals corresponding to each mode that propagates through the waveguide. Then the envelope of the eigen-signals always has nearly the same form, while the amplitudes of real and imaginary part change with small changes of the system. Every received signal can be expanded into the eigen-signals, and in some cases the resulting modespecific amplitudes may be meaningful enough for measurement of liquid properties just as well. Fig. 7 exemplifies the correspondence between the eigen-signals which were determined by a PCA and the modal weights $w_{i}$ that were calculated with modal expansion and by knowledge of the dispersion relations. There are still some ambiguities in the PCA-results but these can be resolved with the knowledge of the analytical eigen-signals.

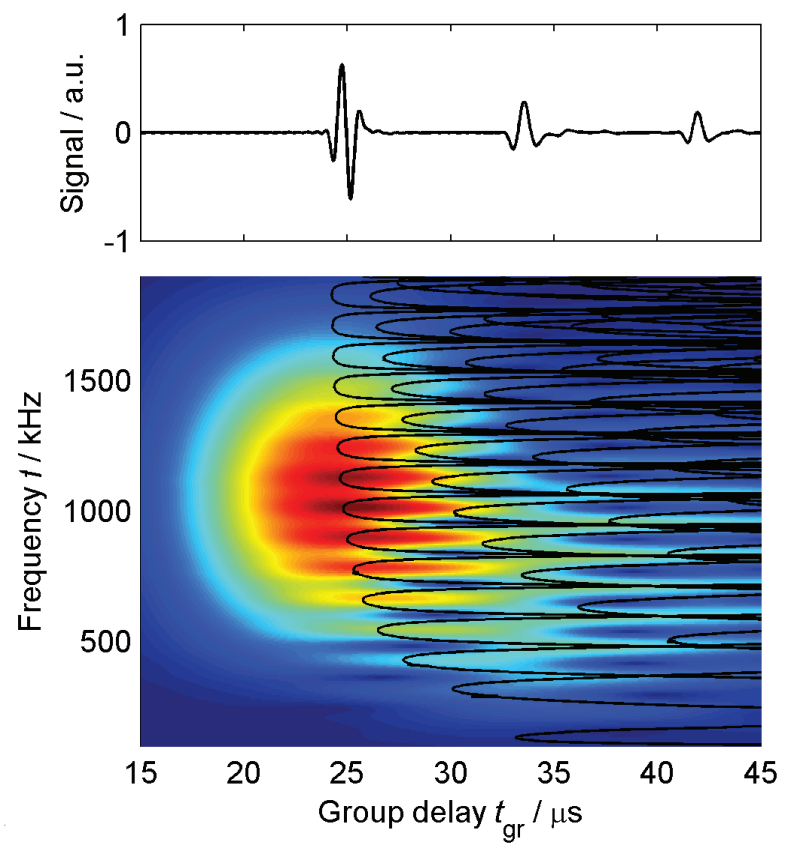

Figure 6: Signal at the end of a $30 \mathrm{~mm}$ long and $6 \mathrm{~mm}$ thick waveguide and the corresponding sonagram; for comparison the group velocities of figure 2 were converted to group delays and plotted as black solid lines in the same figures. 

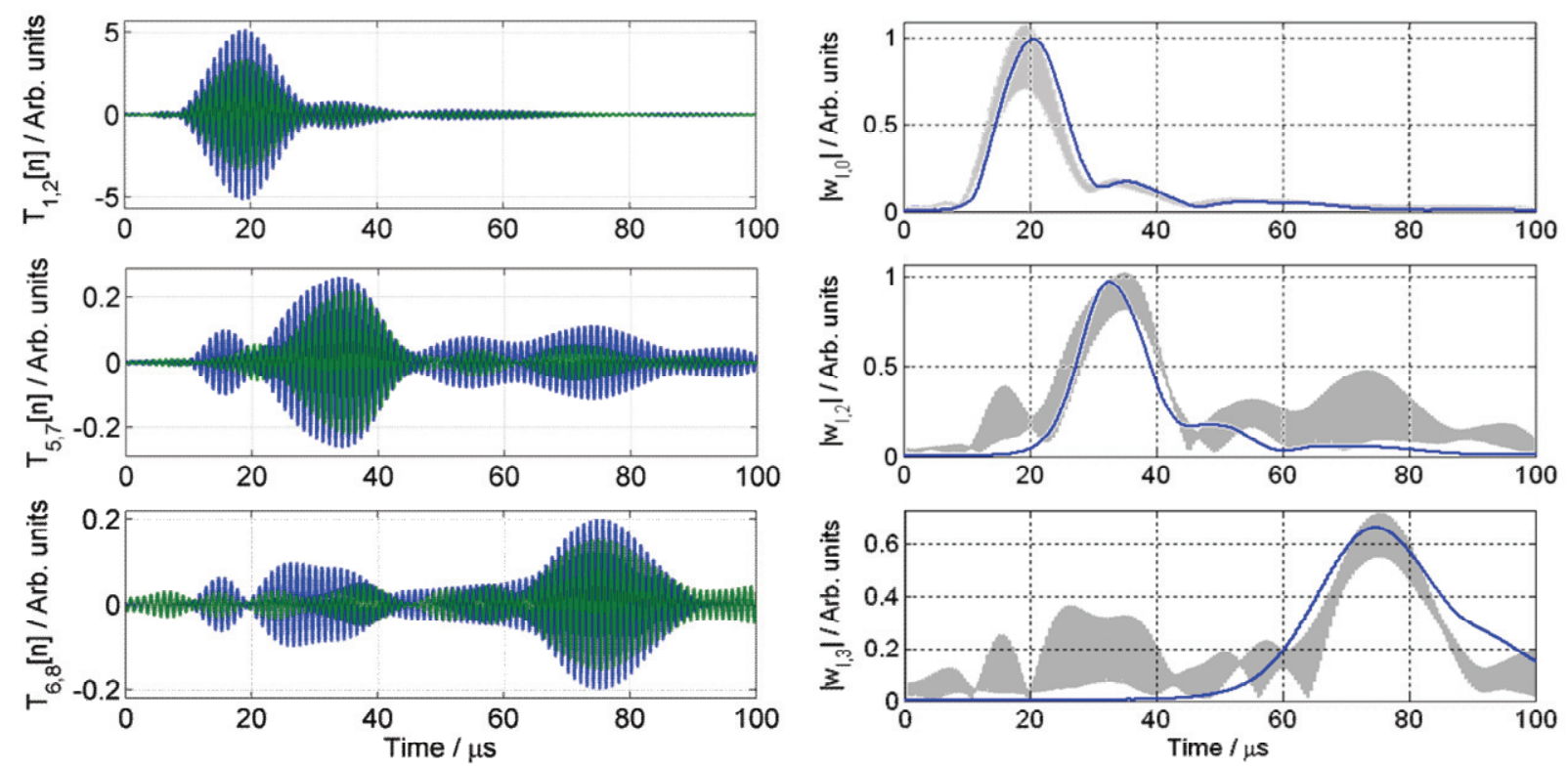

Figure 7: The PCA of different signals at the end of the waveguide results in pairs of eigen-signals (left); the sum of squares of these pairs (right, gray) match the modal weight functions (right, blue)

\section{Conclusion}

The presented methods are suited to model, simulate and measure mode-specific wave propagation in an acoustic waveguide. Taking into acount geometric dispersion these methods are some basic tools for identifying the material specific absorption as well as sound velocity which are interrelated through the local Kramers-Kronig relations. If both functions are known it is also possible to switch to the rheologic properties of the liquid.

\section{References}

[1] V. A. Šutilov: „Physik des Ultraschalls“, Springer-Verlag, Wien 1984, ISBN: 3-211-81798-0

[2] M. O'Donnell, e.a.: "Kramers-Kronig relationship between ultrasonic attenuation and phase velocity", J. of the Acoustical Society of America, Vol. 69 (3), 1981, pp. 696-701

[3] M. Moellendorf, e.a.: „Sensoranordnung für die Ermittlung physikalischer Eigenschaften von Flüssigkeiten“, DE 19850802 A1, 2000

[4] J. Du, e.a.: „A study of Love-wave acoustic sensors“, Sensors and Actuators, A56, 1996, pp. 214

[5] C. Glorieux, e.a.: „On the character of acoustic waves at the interface between hard and soft solids and liquids", J. Acoust. Soc. Am. 110 (3), 2001, pp. 1299-1306

[6] Latifa Qobi, e.a.: „Permeability Determination from Stoneley Waves in the Ara Group Carbonates, Oman”, GeoArabia, Vol. 6 (4), 2001

[7] F. B. Cegla, e.a.: "Material property measurement using the quasi-Scholte mode-A waveguide sensor", J. Acoust. Soc. Am. 117 (3), 2005, pp. 1098-1107

[8] J. McLean and F. Levent Degertekin: "Directional Scholte Wave Generation and Detection Using Interdigital Capacitive Micromachined Ultrasonic Transducers“, IEEE UFFC, 51(6), 2004, pp. 756-764

[9] B. Henning: „Die akustische Impedanz als Messgröße zur Charakterisierung flüssiger Stoffsysteme“, Technisches Messen (71), 2004, S. 492-500

[10] H. Faustmann, e.a.: "Measurement of the properties of liquids based on the dispersion of Lamb waves in an acoustic waveguide", Physics Procedia, Vol. 3 (1), 2010, pp. 959-964

[11] J. Rautenberg and B. Henning: "Simultaneous direct and indirect measurement of sound velocities", Eurosensors XXII, Dresden, 07.-10.09.2008, Proceedings

[12] J. Rautenberg and B. Henning: „New approach for the reliable measurement of the acoustic impedance of liquids in an acoustic waveguide", 13th Intern. Conf. on Sensors, Technologies, Electronics and Applications, Sensor+Test 2007, Proceedings Vol. II, pp. 87-92

[13] G. Lindner, e.a.: "A versatile acoustic waveguide sensor for liquids based on multiple mode conversion at solid-liquid interfaces", Proc. IEEE Ultrasonics Symposium, 2006

[14] M. Maess: "Methods for Efficient Acoustic-Structure Simulation of Piping Systems", Dissertation der Universität Stuttgart, 2006

[15] B. Pavlakovic and M. Lowe: "Disperse - User's Manual”, www.ndt.imperial.ac.uk

[16] F. Bause, e.a.: "An improved mode-tracing algorithm to compute dispersion curves of acoustic waveguides", Proc. IEEE Intern. Ultrasonics Symposium (IUS) 2010

[17] D. Costley: "Dispersion curve analysis of laser-generated Lamb waves", Ultrasonics, Vol. 32 (4), 1994, pp. 249-253 